\title{
Descrição do diagnóstico coletivo de saúde realizado pelo Projeto de Extensão Jornada Universitária da Saúde (JUS) no município de Jambeiro-SP
}

\author{
Description of the collective health diagnosis held by the Extension Project \\ Jornada Universitária da Saúde (JUS) in the city of Jambeiro-SP
}

\author{
Abraão Deyvid Alves de Lima Barreto ${ }^{1}$, Bárbara Castro Possidente ${ }^{2}$, Daniel Baffini de Paula ${ }^{3}$, \\ Gabriela Rigote ${ }^{4}$, Gessika Costa Bueno ${ }^{5}$, Karen Sayuri Kameyama ${ }^{6}$, \\ Priscila Florentino $^{7}$, Nayara de Castro Pereira ${ }^{8}$
}

Barreto ADAL, Possidente BC, Paula DB, Rigote G, Bueno GC, Kameyama KS, Florentino P, Pereira NC. Descrição do diagnóstico coletivo de saúde realizado pelo Projeto de extensão Jornada Universitária da Saúde (JUS) no município de Jambeiro-SP/Description of the collective health diagnosis held by the extension project Jornada Universitária da Saude (JUS) in the city of Jambeiro-SP. Rev Med (São Paulo). 2015 abr.-jun.;94(2):99-105.

\begin{abstract}
RESUMO: O diagnóstico coletivo de saúde, proposto pelo modelo de planejamento normativo de ações em saúde como a etapa inicial, é parte essencial no processo de elaboração e execução de intervenções [1], que visem acolher necessidades de saúde da população de determinada localidade. Entretanto, o conhecimento necessário para a realização de diagnósticos coletivos de saúde nem sempre é trabalhado de maneira adequada e efetiva durante a formação de profissionais da saúde. Essa lacuna do ensino pode ser suprida por atividades de extensão acadêmica, tais como a Jornada Universitária da Saúde (JUS). A JUS é um projeto interprofissional e interdisciplinar de extensão da Universidade de São Paulo, que objetiva a promoção e educação em saúde mediante ações desenvolvidas em uma cidade do interior paulista, em um ciclo trienal. O presente trabalho tem como principal objetivo descrever o primeiro ano da JUS em uma nova cidade de atuação, Jambeiro, apresentando o processo realizado para o macro diagnóstico coletivo de saúde desse município.
\end{abstract}

DESCRITORES: Equipe de assistência ao paciente; Pacotes de assistência ao paciente; Pessoal de saúde; Promoção da Saúde; Educação em saúde; Capacitação de recursos humanos em saúde.

\begin{abstract}
The collective diagnosis in health, proposed by the normative planning model as the initial step, is an essential part in developing and implementing interventions ${ }^{1}$, whose purpose is to receive demands from a specific place. Although essential, the necessary knowledge for conducting collective diagnoses in health is not always taught properly and effectively during professional's health graduation. This gap can be filled with academics extensions, as well as the project "Jornada Universitária da Saúde" (JUS). The JUS is an interprofessional and interdisciplinary project from the University of São Paulo that aims the promotion and education in health through actions that are implemented in an inner city in São Paulo state, on a three year cycle. This article has as it is main goal, describing the project's first year in a new city named Jambeiro, presenting the process of accomplishment for the collective diagnosis in health from this city.
\end{abstract}

KEYWORDS: Patient care team; Patient care bundles; Health personnel; Health promotion; Health education; Health human resource training.

1. Acadêmico do $4^{\circ}$ ano do curso de Medicina da Faculdade de Medicina da Universidade de São Paulo.

2. Acadêmica do $3^{\circ}$ ano do curso de Fisioterapia da Faculdade de Medicina da Universidade de São Paulo.

3. Acadêmico do $3^{\circ}$ ano do curso de Fisioterapia da Faculdade de Medicina da Universidade de São Paulo.

4. Acadêmica do $4^{\circ}$ ano do curso de Nutrição da Faculdade de Saúde Pública da Universidade de São Paulo.

5. Acadêmica do $4^{\circ}$ ano do curso de Fisioterapia da Faculdade de Medicina da Universidade de São Paulo.

6. Nutricionista graduada pela Faculdade de Saúde Pública da Universidade de São Paulo.

7. Acadêmica do $4^{\circ}$ ano do curso de Terapia Ocupacional da Faculdade de Medicina da Universidade de São Paulo.

8. Acadêmica do $4^{\circ}$ ano do curso de Enfermagem da Faculdade de Enfermagem da Universidade de São Paulo.

Endereço para correspondência: Abraão Deyvid Alves de Lima Barreto. Rua Doutor José Amaral, 211- São Paulo, SP, CEP 036750-

10. e-mail: abraaousp100@gmail.com. 


\section{INTRODUÇÃO}

$\mathrm{E}$ m meados do século XX, Leavell e Clark propuseram o modelo da História Natural das Doenças e os diferentes níveis de prevenção. Neste modelo, o conceito de promoção da saúde é uma forma de prevenção primária, que antecede a instalação das doenças ${ }^{2}$. Desde a Conferência de Ottawa (1986), fala-se em uma Nova Promoção da Saúde (NPS), que amplia as práticas promotoras de saúde a partir do conceito ampliado de saúde e será descrita a seguir.

Ao definir o conceito tradicional da promoção de saúde como sendo "práticas destinadas a desenvolver uma saúde ótima" e levando em consideração que atualmente a Organização Mundial de Saúde define como princípios da "nova promoção em saúde" a concepção holística de saúde, a intersetorialidade, o empoderamento, a participação social, a equidade, a multiestratégias e a sustentabilidade é evidente a importância da intensa abordagem desse tema durante a formação acadêmica dos estudantes da área de saúde ${ }^{3}$.

Contudo, os avanços conceituais ainda enfrentam barreiras para sua efetiva implementação. Tais desafios impulsionaram a formulação de políticas públicas que visam auxiliar a fundamentação da promoção da saúde na prática profissional, principalmente no que se refere à Atenção Primária.

No Brasil, a Política Nacional de Promoção de Saúde (PNPS) estabelecida em 2006 discute a educação permanente nas formações da área da saúde, aborda o desafio da formação de profissionais aptos para atuar com a perspectiva da promoção e educação em saúde no Sistema Único de Saúde (SUS).

Ademais, baseando-se na concepção de que "Não se cuida efetivamente de indivíduos sem cuidar de populações, e "Não há verdadeira saúde pública que não passe por um atento cuidado de cada um de seus sujeitos"4 não há meio de elaborar medidas promotoras de saúde sem um efetivo diagnóstico coletivo de saúde da população alvo das intervenções. Este, assim como o diagnóstico na clínica, é a síntese do levantamento de sinais e sintomas que fornecerá conhecimento e informações para ampliar a capacidade de atuar sobre os problemas existentes, orientando quais ações e abordagens serão mais efetivas 5 .

Embora incontestável a importância do diagnóstico coletivo de saúde, nem sempre este é abordado de forma efetiva durante as graduações da área da saúde. Esta lacuna do ensino pode ser minimizada a partir da participação em algumas atividades acadêmicas, como as extensões universitárias.

A extensão universitária é o processo educativo, cultural e científico que articula o ensino e a pesquisa de forma indissociável e viabiliza a relação transformadora entre a universidade e a sociedade (...). Este fluxo, que estabelece a troca de saberes sistematizados/acadêmico e popular, terá como consequência: a produção do conhecimento resultante do confronto com a realidade brasileira e regional; a democratização do conhecimento acadêmico e a participação efetiva da comunidade na atuação da universidade. Além da instrumentalizadora deste processo dialético de teoria/prática, a extensão é um trabalho interdisciplinar que favorece a visão integrada do social ${ }^{6}$.

A Jornada Universitária da Saúde (JUS) é um projeto interprofissional, que conta com graduandos de diferentes semestres dos cursos de Enfermagem, Fisioterapia, Fonoaudiologia, Medicina, Nutrição, Saúde Pública e Terapia Ocupacional da Universidade de São Paulo. Esses estudantes interagem ao longo do ano, para o desenvolvimento de práticas que buscam melhorar a qualidade de vida de determinada população de uma cidade do interior paulista, sobretudo em relação à promoção e educação em saúde.

Visando uma melhor gestão do projeto, os jornadeiros (nome pelo qual os estudantes participantes são chamados) são divididos em grupos específicos, de acordo com as demandas da cidade, a fim de desenvolver intervenções mais direcionadas e eficientes a cada parcela da população. Há ainda a realização de Campanhas da Saúde e Visitas Domiciliares, ambas com enfoque de promoção e educação em saúde.

A cidade de atuação do projeto é escolhida com base em análise de dados socioeconômicos, grau de interesse e disponibilidade da prefeitura municipal. Uma mesma cidade é visitada por três anos consecutivos, cumprindo as seguintes etapas:

$-1^{\circ}$ ano: identificar os problemas locais, destacando as principais necessidades e potencialidades da população, realizando assim um diagnóstico de saúde que possibilite a promoção da saúde;

- $2^{\circ}$ ano: intervir, baseando-se na análise do diagnóstico de saúde obtido pelo ano anterior, com foco nos agentes multiplicadores a fim de tornar o projeto sustentável na cidade;

$-3^{\circ}$ ano: avaliar os resultados e propor planos para melhorar o sistema de saúde e melhorar a qualidade de vida da população.

Em 2013, a JUS iniciou um novo ciclo de atividades na cidade de Jambeiro, interior paulista. O presente artigo tem como objetivo geral descrever o processo realizado para o macrodiagnóstico coletivo de saúde desse município, sendo os objetivos específicos:

1.Descrever como é realizada a escolha da cidade atendida pelo projeto;

2.Descrever como os acadêmicos envolvidos no projeto desenvolveram seus próprios instrumentos para realizar o diagnóstico de saúde;

3.Relatar o aprendizado dos jornadeiros, levando em consideração o trabalho interprofissional para a atuação de promoção e educação em saúde, bem como para a realização de diagnóstico coletivo de saúde. 


\section{MATERIAIS E MÉTODOS}

Trata-se de um relato de experiência, críticoreflexivo. A fim de facilitar a compreensão do processo, a análise é apresentada a partir de dois aspectos: a escolha do município de Jambeiro e a construção do diagnóstico de saúde em si.

\section{A escolha do município de Jambeiro}

A escolha da cidade para o desenvolvimento do projeto é feita a partir da análise de dados em sistemas de informação nacionais, como o DATASUS (Departamento de Informática do SUS) e PNUD (Programa das Nações Unidas para o Desenvolvimento), e posterior visita ao município.

Pelo site do Programa das Nações Unidas (PNUD) acessamos o Atlas do Desenvolvimento Humano no Brasil 2013, que foi fundamental no início do processo de escolha da cidade, com o fornecimento de dados como: população total; IDHM (Índice de Desenvolvimento Humano Municipal) - que permite a caracterização do desenvolvimento socioeconômico do município a partir das renda, longevidade e educação; urbanização; estrutura etária; o coeficiente de GINI; condição de moradia; e mortalidade infantil, para que fosse realizado um macro diagnóstico de saúde do município de Jambeiro.

Já o banco de dados DATASUS foi utilizado para a obtenção de informações para subsidiar as decisões tomadas ao longo do desenvolvimento do projeto, como: número de profissionais de saúde por habitante, proporção da população servida por rede de abastecimento de água, proporção da população servida por esgotamento sanitário, proporção da população servida por coleta de lixo, taxa de mortalidade específica por doenças do aparelho circulatório, e taxa de mortalidade específica por diabetes melito.

Para a tomada de decisão da escolha da cidade, consideramos:

a) o número total da população, já que a cidade a ser escolhida não deveria exceder seis mil habitantes, para não comprometer o desenvolvimento das atividades, pois o número de estudantes envolvidos é finito;

b) privilegiam-se cidades com IDHM mais baixos;

c) urbanização, já que é preciso saber se a cidade tem a infraestrutura necessária para receber toda a equipe do projeto.

A estrutura etária foi utilizada para caracterizar a população, para posterior formação de grupos populacionais, focos das atividades do projeto. O coeficiente de GINI também foi essencial, pois apresenta a possível vulnerabilidade e inequidade econômica da população. A condição de moradia também se fez importante no delineamento socioeconômico. A mortalidade infantil foi considerada um indicador fundamental, uma vez que mede, mesmo que de forma indireta, a qualidade da saúde do município.

O número de profissionais de saúde por habitante possibilitou um maior conhecimento sobre a disponibilidade de profissionais da área de saúde para atender as demandas da população, o que permitiu conhecer sobre os serviços relacionados à saúde e oferecidos pela cidade aos seus habitantes. Os indicadores de proporção da população servida por rede de abastecimento de água, proporção da população servida por esgotamento sanitário e proporção da população servida por coleta de lixo foram importantes para o conhecimento acerca da condição em que os moradores do município a ser escolhido viviam, ou seja, sobre o saneamento básico.

Por fim, os indicadores de taxa de mortalidade específica por doenças do aparelho circulatório e taxa de mortalidade específica por diabetes melito foram de grande importância, pois revelam a condição geral de vida e de saúde da população em determinado lugar, além de relatar as peculiaridades que contribuíram para uma morte avaliando assim, de forma indireta, a qualidade da saúde do município ${ }^{1}$.

Com os diagnósticos coletivos em saúde embasados nos dados epidemiológicos levantados no PNUD e DATASUS, enviamos um e-mail apresentando o projeto e demonstrando interesse em sua realização no município. Após resposta da prefeitura, foram agendadas viagens para o conhecimento do território e início do diálogo com gestores da cidade, para abordar e avaliar os seguintes aspectos: grau de interesse do município em receber a JUS, disponibilidade de recursos para fornecer a contrapartida durante nossa expedição (alojamento, alimentação, segurança e transporte) e a possibilidade de criação de parcerias com profissionais e equipamentos sociais do município, com intuito de garantir auxílio na realização de nossas atividades e intervenções.

Após acordo, entre a prefeitura e coordenadores do projeto, o município de Jambeiro foi escolhido para o desenvolvimento da Jornada Universitária da Saúde, nos anos de 2013 a 2015.

\section{O Diagnóstico em Saúde realizado pelas ações da JUS}

O Diagnóstico em Saúde do município é realizado a partir de ações, como a campanha de saúde, visitas domiciliares e intervenções coletivas, realizadas pelos grupos populacionais específicos. Dessa maneira, conseguimos englobar, outros aspectos importantes à análise, além do processo saúde-doença, ecológicos, sociopolíticos, demográficos e dos recursos disponíveis?

De maneira sucinta, descrevemos a metodologia das visitas domiciliares, da campanha de saúde bem como ações de intervenções coletivas (realizadas pelos grupos populacionais), com o propósito de detalhar o diagnóstico de saúde dos munícipes atendidos pelo projeto. 


\section{a) Visitas Domiciliares}

Com o intuito de promover educação em saúde, as (VD's) foram selecionadas e realizadas com o apoio das Agentes Comunitárias de Saúde (ACS's) da cidade. Cada visita contou com a participação de dois estudantes de diferentes cursos. Realizaram-se duas visitas por residência, na qual a primeira tinha objetivo de conhecer a realidade da família, aplicando um questionário elaborado pelos próprios alunos a partir de questionários pré-existentes, como o utilizado pelo VIGITEL.

Constituído por 45 questões, o instrumento engloba temas como: caracterização socioeconômica dos residentes, doenças referidas, tratamento de doenças, sintomas referidos, saúde do homem, saúde da mulher, perfil do ambiente, alimentação, autopercepção de saúde, hábitos, motivação para a mudança e atividades de lazer.

Em posse das respostas obtidas na primeira visita, a dupla de jornadeiros discutia e elaborava possíveis orientações para a família, ponderando os conteúdos e a melhor forma de abordagem. A segunda VD buscou discutir (e quando possível orientar) necessidades levantadas. Ao término da segunda VD, os questionários foram entregues aos estudantes coordenadores do projeto, que posteriormente tabularam os dados e os analisaram com o auxílio do programa STATA versão 13.0.

\section{b) Campanha da Saúde}

Aberta à todos os munícipes, a Campanha da Saúde foi um evento com o objetivo de realizar exames de rastreamento e, principalmente, desenvolver educação em saúde. Para tanto, em Jambeiro os jornadeiros realizaram os exames de glicemia capilar, aferição de pressão arterial e antropometria.

Considerando a oportunidade educativa do encontro com os munícipes, foram confeccionadas mesas lúdicas com analogias que visavam facilitar a compreensão dos usuários sobre os seguintes temas: Saúde do trabalhador, Diabetes Mellitus, Hipertensão Arterial Sistêmica e Hipercolesterolemia.

Cabe aqui reforçar que as ações da campanha eram iniciadas e direcionadas, a partir de uma ficha de triagem ao usuário. Esta foi elaborada pelos estudantes, e contava com 20 questões sobre: caracterização do indivíduo, Diabetes Mellitus, Hipertensão Arterial, Saúde do trabalhador e auto avaliação da saúde. Os dados obtidos pela ficha de triagem foram tabulados e posteriormente analisados com o auxílio do programa STATA versão 13.0.

\section{c) Intervenções coletivas}

Em 2013, os 75 participantes do projeto foram divididos em grupos populacionais de trabalho, a saber: Grupo Crianças, Grupo Adolescentes, Grupo Escola, Grupo Posto (Unidade Básica de Saúde) e Grupo Social.
A população alvo de cada grupo é ilustrada no Quadro 1.

Quadro 1 - Grupos de trabalho e seu público alvo. Jornada
Universitária da Saúde, Jambeiro - 2013
\begin{tabular}{|l|l|}
\hline Grupo & Público alvo \\
\hline Adolescentes & $\begin{array}{l}\text { Estudantes do } 9^{\circ} \text { ano do ensino fundamental } \\
\text { até o } 3^{\circ} \text { ano do ensino médio. }\end{array}$ \\
\hline Crianças & $\begin{array}{l}\text { Alunos do } 1^{\circ} \text { ao } 5^{\circ} \text { ano do ensino } \\
\text { fundamental. }\end{array}$ \\
\hline Escola & $\begin{array}{l}\text { Alunos, pais, professores e comunidade } \\
\text { escolar. }\end{array}$ \\
\hline Posto (UBS) & $\begin{array}{l}\text { Agentes Comunitárias da Saúde, } \\
\text { funcionários da UBS, gestantes e idosos. }\end{array}$ \\
\hline Social & População geral \\
\hline
\end{tabular}

Com o propósito de promover uma participação conjunta das diferentes profissões na elaboração de atividades de intervenção coletiva que visem promoção e educação em saúde, assim como o rastreamento de possíveis demandas da população alvo, os jornadeiros foram divididos de modo que cada grupo possuía, ao menos, dois integrantes de cada curso. Essa divisão é essencial ao projeto, visto que tomamos por ponto de partida que o campo da saúde possui questões que não se restringem a uma única profissão ou disciplina, e, portanto, exige a articulação de diferentes saberes, conhecimentos e práticas ${ }^{8}$.

Após a formação dos grupos, as reuniões ocorreram no dia e horário mais adequado à maioria dos membros. Durante as primeiras reuniões, buscou-se o entrosamento da equipe para que as discussões e ideias pudessem surgir mais facilmente no decorrer dos próximos encontros. Ademais, nessas reuniões foram traçados os planos de ação, em que se analisou o público alvo, as intervenções, os locais e os objetivos e prioridades das ações planejadas.

De forma geral, cada grupo se reuniu de 10 a 12 vezes, sendo que cada encontro durava aproximadamente duas horas. É importante frisar que a confecção de atividades de intervenção coletivas ocorreu a partir da comunicação e colaboração entre os membros da equipe, com interação e troca de conhecimentos entre si. Fica claro então, que as práticas da JUS extrapolam a justaposição de especialidades de uma equipe multiprofissional, adentrando em ações que geram o conhecimento de maneira coletiva, interprofissional e interdisciplinar.

Assim como é feito no modelo de planejamento normativo nos serviços de saúde, que propõe o diagnóstico de saúde como etapa inicial para a elaboração das intervenções futuras ${ }^{1}$, os grupos do projeto tiveram como principal objetivo nesse primeiro ano de atuação em Jambeiro, conhecer a realidade, as necessidades e potencialidades de sua população alvo. 


\section{RESULTADOS}

\section{A escolha do município de Jambeiro}

Os dados do Quadro 2 ilustram os resultados obtidos sobre Jambeiro na pesquisa realizada no Atlas do Desenvolvimento Humano no Brasil 2013, que nos forneceu dados fundamentais para a escolha do município. A população de 5.349 habitantes, assim como o grau de urbanização, mostraram-se adequados para a realização do projeto. Ao analisar os outros indicadores, como o índice GINI, IDHM, condição de moradia e mortalidade infantil, nota-se que Jambeiro possui condições socioeconômicas e de saúde boas, dentro do contexto brasileiro.

Quadro 2 - Dados de Jambeiro obtidos no Atlas de Desenvolvimento Humano de 2013

\begin{tabular}{|c|c|c|c|}
\hline População Total & \multicolumn{3}{|l|}{5.349 habitantes } \\
\hline IDHM & \multicolumn{3}{|r|}{0,756} \\
\hline \% de Taxa de Urbanização & \multicolumn{3}{|r|}{47,88} \\
\hline \multirow{2}{*}{ Estrutura etária } & $<15$ anos & $15-64$ anos & $>65$ anos \\
\hline & 1.233 & 3.684 & 432 \\
\hline $\begin{array}{l}\text { Condição de Moradia } \\
\text { (\% de pessoas em domicílios com abastecimento de } \\
\text { água e esgotamento sanitário inadequados) }\end{array}$ & \multicolumn{3}{|r|}{0,28} \\
\hline Mortalidade Infantil (por 1.000 nascidos vivos) & \multicolumn{3}{|r|}{12,4} \\
\hline
\end{tabular}

Os dados dos indicadores, listados no Quadro 2, demonstram que Jambeiro apresentava condições de oferecer o suporte necessário para que as atividades da JUS fossem implementadas de maneira abrangente e eficiente, concedendo, acima de tudo, uma sólida base que pode ser explorada, a fim de que as ações do projeto tenham continuidade no município, mesmo após o fim do ciclo trienal.

Embora tenha sido de grande valia toda a coleta de dados no Atlas de Desenvolvimento Humano no Brasil 2013, o fator determinante para a escolha de Jambeiro como alvo das ações da JUS durante o ciclo 2013-2015, foi o interesse demonstrado pelo município em receber o projeto. As reuniões com os gestores foram ótimas oportunidades para a criação de vínculo entre a cidade e a JUS. Além disso, nessas reuniões foram levantadas as necessidades do município passíveis de intervenção. Diversas demandas foram relatadas durante os encontros entre os gestores de Jambeiro e os alunos coordenadores do projeto. Dentre elas, destacaram-se a pequena utilização da praça pelos jovens da cidade, gravidez, uso de drogas pelos adolescentes e recursos da UBS nem sempre utilizados de maneira adequada pelos usuários.

Esse levantamento de demandas foi somado à análise de dados no DATASUS e nos forneceu a base para a realização de nossas ações no município. O Quadro 3 apresenta indicadores obtidos nesse banco de dados, os quais julgamos primordiais para a definição de nossas prioridades e objetivos durante o primeiro ano de atuação da JUS no município.

Quadro 3 - Dados de Jambeiro obtidos no DATASUS.

\begin{tabular}{|c|c|}
\hline \multicolumn{2}{|c|}{ A cada 1000 habitantes } \\
\hline Número de profissionais de saúde & 3 \\
\hline \multicolumn{2}{|c|}{ Proporção de Moradores por Tipo de Abastecimento de Água, Instalação Sanitária e Coleta de Lixo (\%) } \\
\hline Abastecimento de Água & 51,6 \\
\hline Esgotamento Sanitário & 46,7 \\
\hline Coleta de Lixo & 81,3 \\
\hline \multicolumn{2}{|c|}{ Coeficiente de Mortalidade para algumas causas selecionadas (por 100.000 habitantes) } \\
\hline $\begin{array}{l}\text { Mortalidade específica por doenças do aparelho } \\
\text { circulatório }\end{array}$ & 36,4 \\
\hline Mortalidade específica por Diabete Mellitus & 18,5 \\
\hline
\end{tabular}




\section{O diagnóstico em saúde realizado pelas ações da JUS}

Todas as ações da JUS em Jambeiro basearam-se no levantamento dos quadros de saúde e socioeconômico, obtidos durante o processo de escolha do município como cidade oficial do ciclo 2013-2015. Os resultados apresentados a seguir se baseiam em relatos dos jornadeiros, relatórios pós atividades, análise dos dados dos questionários aplicados durante as Visitas Domiciliares e Campanha de Saúde.

É importante ressaltar que nossas ações não visavam, necessariamente, a realização do diagnóstico da realidade de todos os munícipes de Jambeiro, mas sim um levantamento das demandas, focado nos indivíduos que foram alvo das intervenções da JUS em 2013 e que serão, possivelmente, a população que participará de nossas ações em 2014 e 2015. Não nos restringimos à análise dos resultados do presente artigo em número, como dados quantitativos, mas principalmente como demandas diagnosticadas por nossas ações.

Visitamos 65 domicílios durante nossas visitas domiciliares, abrangendo um total de 225 moradores. A maioria dos domicílios visitados são compostos de 3 a 5 residentes, com renda referida de 3 a 5 salários mínimos. Identificamos casos de abuso de álcool, uso inadequado de remédios e fumantes que desejam largar o vício. Ademais, foi alta a frequência de sintomas relatados, com destaque para a grande quantidade de casos de dor crônica e dificuldade para se enxergar. As questões sobre o ambiente revelaram baixa frequência de pessoas que reciclam o lixo, e problemas relacionados à limpeza da caixa d'agua adequada.

Atendemos 143 munícipes em nossa Campanha da Saúde. Durante o evento foi percebido um grande interesse em aprender e tirar dúvidas sobre os temas abordados, pela maioria da população. Uma parcela significativa dos usuários avaliou sua saúde como sendo regular ou ruim, mesmo sem doenças diagnosticadas. Houve, ainda, alta prevalência de casos referidos de Hipertensão, Hipercolesterolemia e Diabete Mellitus por parte dos munícipes que, por sua vez, se tratavam majoritariamente no sistema público do próprio município.

Assim como as Campanhas da Saúde e VD's, as intervenções de saúde coletivas demonstraram ser excelentes oportunidades para a realização de um rastreamento efetivo das demandas da população. A partir das atividades realizadas pelos grupos populacionais, foram identificadas demandas; assim, os principais assuntos/temas que devem abordados nos anos posteriores de ação da JUS estão ilustrados no Quadro 4.

Todos os dados obtidos por meio das ações da JUS em 2013 estão sendo organizados e reunidos num documento. Este será entregue tanto para os gestores de Jambeiro quanto para a nova coordenação do projeto. Intitulado de "Relatório Final", esse arquivo é a base para a atuação do projeto nos próximos anos do ciclo, objetivando uma atuação conjunta, por meio de práticas comunicativas e colaborativas, entre a JUS e o município.

Quadro 4 - Assuntos/temas identificados pelos grupos populacionais como demandas de trabalho, em suas ações de promoção e educação em saúde

\begin{tabular}{|l|l|}
\hline Grupo & Assuntos/temas \\
\hline Adolescentes & $\begin{array}{l}\text { Gravidez na adolescência } \\
\text { Uso de álcool e drogas } \\
\text { Ócio }\end{array}$ \\
\hline Crianças & $\begin{array}{l}\text { Maior dinamismo nas atividades } \\
\text { Alimentação saudável e profissões }\end{array}$ \\
\hline Escola & $\begin{array}{l}\text { Uso de drogas e sexualidade com os } \\
\text { adolescentes } \\
\text { Preparo dos professores para abordar tais } \\
\text { temas }\end{array}$ \\
\hline Posto (UBS) & $\begin{array}{l}\text { Áreas afastadas do centro que carecem } \\
\text { de educações educativas e promotoras de } \\
\text { saúde. }\end{array}$ \\
\hline Social & $\begin{array}{l}\text { Agressões verbais entre os alunos, } \\
\text { incluindo bullying }\end{array}$ \\
\hline
\end{tabular}

\section{DISCUSSÃO}

Durante a formação profissional, os projetos de extensão, tais como a JUS, proporcionam um conhecimento enriquecedor ao indivíduo, pois permitem o contato do acadêmico com a população e com a realidade, sendo, muitas vezes, realidades distintas das suas, situações únicas. A expansão do processo ensino-aprendizagem incluindo cenários diferenciados para a formação, rompe o modelo atual muitas vezes restrito à reprodução do conhecimento.

Assim, é possível verificar a teoria vista em sala de aula na prática, aprimorando a sua formação. Esses projetos têm como um dos objetivos principais contribuir para a formação de um profissional diferenciado, capaz de compreender o ser humano como um todo, apto a solucionar problemas e a trabalhar de forma interdisciplinar, respeitando o trabalho do outro. Essa dinâmica de atuação dos jornadeiros permite ainda que os estudantes se atentem às necessidades do outro, transcendendo a sua prática profissional, contribuindo para uma formação mais humanizada e não apenas profissional, mas também como indivíduo.

Além dos estudantes, a população da cidade também é beneficiada com a realização das atividades educativas e lúdicas, que têm o objetivo de estimular a promoção da saúde e apresentar a sua importância para o cotidiano das pessoas. Isto é, proporcionar melhoria na qualidade de 
vida dos moradores da cidade, de modo que esses hábitos e práticas sejam multiplicados, ou seja, cultivados, ensinados e capazes de atingir grande parte da população. Assim, a partir desse trabalho interdisciplinar, o diagnóstico em saúde realizado no $1^{\circ}$ ano da JUS no município apresenta um caráter amplo e rico, pois é resultado das ações efetuadas conjuntamente por estudantes de diversos cursos, com diferentes formações.

Desse modo, o diagnóstico em saúde realizado, com intuito de construir um panorama de necessidades e soluções, permite a identificação e caracterização da realidade apresentada. O conhecimento dessa situação de saúde do município permite a obtenção de um diagnóstico fundamental para a melhoria das ações de saúde desenvolvidas pelo projeto, pois disponibiliza informações que podem auxiliar no planejamento das atividades nos anos posteriores. Assim, as ações podem ser estruturadas a partir do que foi diagnosticado no primeiro ano, de acordo com a realidade local.

\section{CONCLUSÃO}

A partir da descrição do primeiro ano da JUS em Jambeiro, foi evidenciado que o processo de diagnóstico coletivo de saúde do município é parte essencial no processo de elaboração e execução de intervenções que visam acolher as necessidades da população. Assim, o trabalho realizado nos anos posteriores é otimizado, uma vez que é planejado de acordo com o diagnóstico detalhado realizado no primeiro ano.

Além disso, verificou-se que a JUS permite ao acadêmico a oportunidade de trabalhar com a população e contribui para que seja formado um cidadão diferenciado, capaz de visualizar um indivíduo como um todo e de realizar práticas colaborativas em prol da população. Assim, a Jornada Universitária da Saúde atua como um importante instrumento na formação de profissionais aptos a atuar com a perspectiva da Promoção da Saúde junto ao Sistema Único de Saúde.

Agradecimentos: Agradecemos todas as pessoas envolvidas no planejamento e execução da Jornada Universitária da Saúde no ano de 2013: jornadeiros, coordenadores, gestores de saúde de Jambeiro e professores orientadores. Em especial, gostaríamos de prestar agradecimentos à Prof. Doutora Ana Claudia C. G. Germani, do departamento de Preventiva da Faculdade de Medicina da Universidade de São Paulo, cuja contribuição ao projeto, assim como para esse trabalho foi imensurável. Obrigado a todos, sem vocês nada disso seria possível.

\section{REFERÊNCIAS}

1. Drumond Junior M. Epidemiologia em serviços de saúde: conceitos, instrumentos e modos de fazer. In: Campos GWS, Minayo MCS, Akerman M, Drumond Júnior M, Carvalho YM. Tratado de saúde coletiva. Rio de Janeiro: Hucitec/ Fiocruz; 2006. p.419-56. (Saúde em debate, 170).

2. Leavell S, Clark EG. Medicina preventiva. São Paulo: McGraw-Hill; 1976.

3. World Health Organization. Health promotion evaluation: recommendations to policymakers. Copenhagen: European Working Group on Health Promotion Evaluation; 1998. Available from: http://apps.who.int/iris/ bitstream/10665/108116/1/E60706.pdf.

4. Ayres JRCM. O cuidado, os modos de ser (do) humano e as práticas de saúde. Saude Soc (São Paulo). 2004;13(3):16-29. http://dx.doi.org/10.1590/S0104-12902004000300003.

5. Vaughan JP, Morrow RH. Epidemiologia para os municípios: manual para o gerenciamento dos distritos sanitários: Hucitec; 1992.

6. I Encontro de Pró-Reitores de Extensão das Universidades Públicas Brasileiras, Brasília; 4-5 nov. 1987. Conceito de extensão, institucionalização e financiamento. Brasília: UNB; 1987. Disponível em: http://www.renex.org.br/ documentos/Encontro-Nacional/1987-I-Encontro-Nacionaldo-FORPROEX.pdf.

7. Pereira MG. Epidemiologia: teoria e prática. Rio de Janeiro: Guanabara-Koogan; 1995.

8. Abrahao AL, Cordeiro BC, Marques D, DAher DV, Teixeira GHMC, Monteiro KA, Gomes LN, Senna MAA, Gouvêa MV, Aboud S, Fernandez VS. A pesquisa como dispositivo para o exercício no PET-Saúde UFF/FMS Niterói. Rev Bras Educ Med. 2011;35(3):435-40. Disponível em: http://www. scielo.br/pdf/rbem/v35n3/a19v35n3.pdf. 